\title{
A RESPONSE BOUND FOR HYSTERETIC SECOND ORDER SYSTEMS*
}

\author{
BY \\ N. MOSTAGHEL \\ University of Utah
}

The behavior of many engineering systems is governed by the second order differential equation

$$
\ddot{u}(t)+f[u(t)]=g(t),
$$

where $g(t)$ is a specified oscillatory function of time, dot denotes differentiation with respect to $t, f(u)$ is a nonlinear restoring function representing the system hysteresis, as shown in the figure, and $u(0)=\dot{u}(0)=0$. To obtain the bound, both sides of equation (1) are multiplied by $\dot{u}$. Integration of the resulting expression over the time interval $t_{i}$ to $t_{i+1}$ yields

$$
\left.\frac{\dot{u}^{2}(t)}{2}\right]_{t_{i}}^{t_{i+1}}+\int_{t_{1}}^{t_{i+1}} f(u) \dot{u}(\tau) d \tau=\int_{t_{i}}^{t_{i+1}} g(\tau) \dot{u} d \tau .
$$

If $t_{i}$ and $t_{i+1}$ are two consecutive times of zero crossing of $\dot{u}(t)$, then the first term on the left hand side of the above equation vanishes. Therefore,

$$
\int_{t_{i}}^{t_{i+1}} f(u) \dot{u}(\tau) d \tau=\int_{t_{i}}^{t_{i+1}} g(\tau) \dot{u}(\tau) d \tau .
$$

Since $u(t)$ attains extremum values at times $t_{i}$ and $t_{i+1}$, then one may assume $\bar{u}=\sup |u(t)|$ to occur either at $t=t_{i}$ or $t=t_{i+1}$. Therefore, the right hand side of equation (3) may be represented by

$$
\int_{t_{i}}^{t_{i+1}} g(\tau) \dot{u}(\tau) d \tau=\int_{u\left(t_{i}\right)}^{u\left(t_{i+1}\right)} g(\tau) d u \leqslant 2 \overline{g u},
$$

where $\bar{g}=\sup |g(t)|$. The left hand side of equation (3) may also be represented as

$$
\int_{t_{i}}^{t_{i+1}} f(u) \dot{u}(\tau) d \tau=\int_{u\left(t_{i}\right)}^{u\left(t_{i+1}\right)} f(u) d u=\alpha(2 \bar{f} \bar{u}),
$$

where $\bar{f}=\sup |f(u)|$ and $0<\alpha \leqslant 1$ is the reduction factor which makes the equality satisfied. The value of $\alpha$ may be calculated from the ratio $A / 4 \bar{f} \bar{u}$, where $A$ is the area of the hysteresis loop. Comparisons of equalities (3) and (5) and inequality (4) yield

$$
\bar{f} \leqslant \bar{g} / \alpha .
$$

\footnotetext{
* Received February 21, 1984.
} 


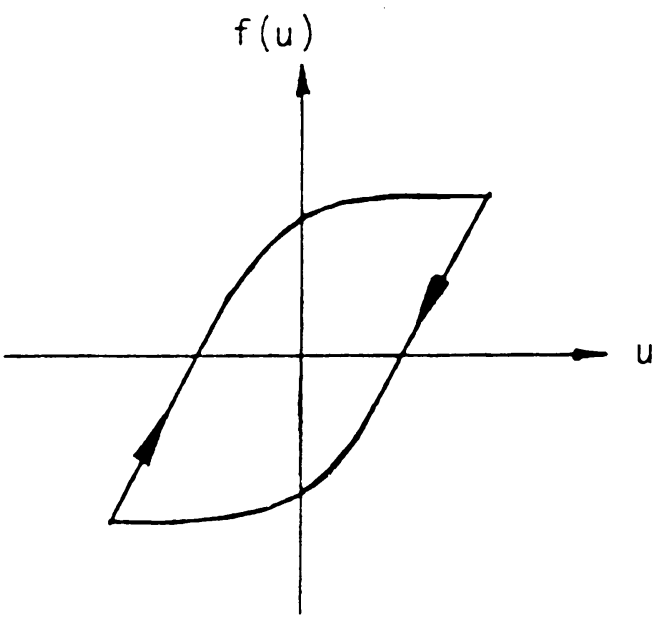

A representation of $f(u)$

If it is assumed that $\bar{u}$ and $\bar{f}$ occur simultaneously, i.e. $\bar{f}=|f(\bar{u})|$, it is concluded that

$$
f(\bar{u}) \leqslant \bar{g} / \alpha .
$$

This inequality yields an upper bound on $\bar{u}$ with $\alpha$ as a parameter. 